\title{
The inhibitory avoidance test optimized for discovery of cognitive enhancers
}

\author{
Eric R. A. Y. Detrait, Étienne Hanon, Bertrand Dardenne, And Yves Lamberty \\ UCB Pharma, Braine-l'Alleud, Belgium
}

\begin{abstract}
In the present article, we describe a new protocol for the inhibitory avoidance test, with a dual purpose: (1) to provide a less variable and more reliable assessment of the efficacy of potential cognitive enhancers in antagonizing scopolamine-induced long-term-memory deficits, and (2) to secure a high throughput for pharmacological screening of cognitive enhancers. The new protocol consists of two acquisition trials that are followed $24 \mathrm{~h}$ later by a single retention trial. In the present study, this protocol clearly dissociated the frequency distributions of retention latencies between scopolamine- and vehicle-treated groups and allowed validation by means of two acetylcholinesterase inhibitors - tacrine and donepezil - that proved to be active in counteracting the scopolamine-induced memory deficit. This protocol also produced stability of the behavioral response to pharmacological agents over a 3-year period. A statistical power analysis indicated that, depending on the efficacy of the drug/dose, a sample size of 5-12 mice was required in order to show a reversal of the scopolamineinduced memory deficit. The double-trial acquisition protocol is suitable for testing cognitive enhancers, while also providing a clearly enhanced throughput.
\end{abstract}

One of the challenges in the pharmacological screening of drugs is to maximize the throughput of behavioral tests in order to evaluate the potential efficacy of large numbers of compounds produced by medicinal chemistry (Brunner, Nestler, \& Leahy, 2002). The maximization of throughput is particularly crucial for in vivo testing of putative cognitive enhancers, since most procedures are time consuming. A single experiment aimed at evaluating a useful range of doses often requires weeks or months. Furthermore, because of interindividual variability, large sample sizes are required in order to allow statistically meaningful conclusions. For these reasons, the in vivo screening for pharmacodynamic efficacy is often the ratelimiting step in the development of a new drug. As a consequence, behavioral testing must be a stepwise process, starting with simple and fast entry tests that should have a higher throughput than do more advanced and complex paradigms. In cognitive behavioral testing, the inhibitory avoidance test-also called "passive avoidance"-is an example of such an entry test in the screening cascade. Inhibitory avoidance has long been used to measure drug effects on long-term memory in rodents (Banfi, Cornelli, Fonio, \& Dorigotti, 1982), to evaluate drug-induced side effects (Kallman \& Condie, 1985), and to assess the efficacy of drugs against scopolamine-induced memory deficits (Dilts \& Berry, 1967; Pan et al., 2006; Ray \& Nagy, 1978). Scopolamine is a nonselective muscarinic receptor antagonist, blocking all four subtypes of postsynaptic receptors as well as the presynaptic M2 autoreceptor, therefore inducing a brain cholinergic dysfunction. The scopolamine-induced cholinergic dysfunction results in memory deficits that model to some extent those observed in Alzheimer's disease (Caulfield, 1993; Ebert \& Kirch, 1998).

The inhibitory avoidance paradigm is based on inhibition of natural photophobia in rodents. Mice are placed in a two-compartment shuttle box that is composed of a bright compartment containing the animal and a dark compartment toward which the animal naturally flees in order to escape from the bright light (see Figure 1). When an animal steps through to the dark compartment, a guillotine door separating the two compartments closes, and a mild nonescapable electrical shock is administered (acquisition trial). Sometime after the sole or last acquisition trial (e.g., $24 \mathrm{~h}$ ), the latency until stepping through toward the dark side is recorded in the absence of an electrical shock (retention trial) and is taken as a measure of long-term memory.

The experimental schemes found in the literature for the inhibitory avoidance paradigm substantially vary in terms of shock intensity, shock duration, and number of trials (Sahgal, 1993). The shock intensity and duration largely vary between study from 0.1 to $4 \mathrm{~mA}$ and from 1 to $10 \mathrm{sec}$ (for a review, see Sarter, Hagan, \& Dudchenko, 1992). Increasing the number of trials or the sample size obviously results in a decreased throughput. The shortest protocol for an inhibitory avoidance test therefore comprises two trials: a single acquisition trial, followed by a single retention trial generally run $24 \mathrm{~h}$ later (Bartus, Dean, Goas, \& Lippa, 1980; Bohdanecký \& Jarvik, 1967; Jarvik \& Kopp, 1967). The downside of this protocol is the large interindividual variability of responses, which implies that a fairly large sample size is usually required to obtain sta-

E. R.A.Y. Detrait, eric.detrait@ucb-group.com 


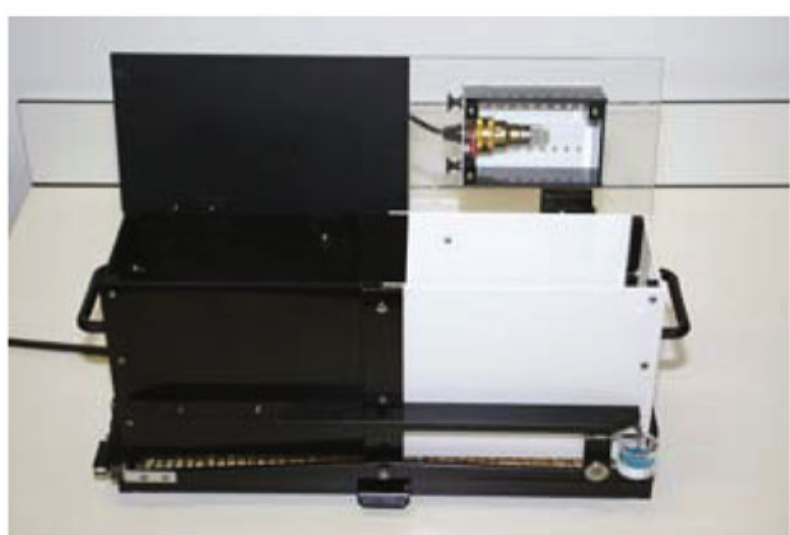

Figure 1. Ugo Basile inhibitory avoidance box.

tistically meaningful information (Elrod \& Buccafusco, 1988; Lamberty \& Gower, 1990). Other commonly used protocols are the multiple-trial acquisition protocols in which successive acquisition trials are run either successively or at a 24-h intertrial interval, and in which a single retention trial is run the last day of testing (Graham \& Buccafusco, 2001; Ray \& Nagy, 1978). The downside of these protocols is the low throughput consequent to the number of acquisition trials.

In the present study, we aimed to decrease the variability and increase the throughput of the inhibitory avoidance test for pharmacological screening of cognitive enhancers. We minimized the number of trials and optimized the scopolamine-induced memory deficit in order to increase the window available for modulating the deficit with pharmacological agents. The single-trial acquisition protocol (one acquisition trial and one retention trial $24 \mathrm{~h}$ later) was improved by adding one acquisition trial that was administered 2 min after the first acquisition trial.

We showed that the double-trial acquisition protocol maximizes the difference between the vehicle- and scopolamine-treated groups by separating their respective frequency distributions. We also showed that such a protocol is sensitive to the effects of two widely used acetylcholinesterase inhibitors against a scopolamine-induced long-term-memory deficit, and that it produces reliable and reproducible results over a long time period, thus allowing a substantial decrease in the sample size required to obtain statistically significant results.

\section{METHOD}

Eight- to 9-week-old male C57Black6J mice (25-30 g) were housed in polypropylene cages under standard housing conditions $\left(22^{\circ} \pm 2^{\circ} \mathrm{C}, 12: 12\right.$-h light:dark cycle, water and food ad lib), to which they were habituated for 2 weeks prior to experimentation. Males were exclusively used in order to avoid the influence of the estrus cycle on drug metabolism and/or efficacy.

All experiments were carried out according to the European guidelines $86 / 609 / \mathrm{CEE}$ and 2003/65/CE and to the Belgian legislation of August 14, 1986, and its amendments.

Tacrine hydrobromide (Sigma), donepezil (Sequoia Research), and scopolamine hydrobromide (Sigma) were diluted in either 1\% methylcellulose $/ 5 \%$ DMSO or $0.9 \% \mathrm{NaCl}$. Vehicle, tacrine, done- pezil, and scopolamine were administered intraperitoneally $30 \mathrm{~min}$ prior to the first acquisition trial.

The Ugo Basile shuttle box (see Figure 1) was divided into two compartments of equal size $(18 \times 10 \times 16 \mathrm{~cm})$ and equipped with a grid floor. One compartment was made of white panels and illuminated with a lamp placed on top of the chamber $(\approx 350$ lux $)$. The other compartment was made of black panels $(\approx 4$ lux $)$. The two compartments were separated by a guillotine door.

The double-trial acquisition protocol consisted of two successive acquisition trials separated by $120 \mathrm{sec}$, which were followed by a single retention trial $24 \mathrm{~h}$ later (Figure 2). After each acquisition trial, mice received a mild electric shock upon stepping through toward the dark side $(0.3 \mathrm{~mA}$ for $3 \mathrm{sec}$, in AC current with a bipolar 10 -msec pulse width and a $20-\mathrm{Hz}$ scrambling frequency between the different bars of the grid floor). This shock intensity was above threshold intensity and was followed by the vocalization of the animals upon receiving the shock. The step-through latency was recorded. Mice failing to step through after $120 \mathrm{sec}$ during the first acquisition trial were excluded from the study. Mice that did not step through after $300 \mathrm{sec}$ during the second acquisition trial were gently pushed to the dark side. Mice that did not step through during the retention trial were assigned a retention latency of $300 \mathrm{sec}$.

The single-trial acquisition protocol was run as usually described in the literature. The mice ran a single acquisition trial during which they received a $0.3-\mathrm{mA}$ electric shock for $3 \mathrm{sec}$ upon stepping through the dark side. The retention trial was run $24 \mathrm{~h}$ later.

Statistical analysis was performed by means of the MannWhitney test, as was required by the non-Gaussian distribution of the data. The results are presented in the following section as medians and first and third quartiles. The power, which is the probability of statistically detecting true differences between groups, was estimated by a bootstrapping method: A collection of samples $(1,000)$ of varying sizes (from 5 to 80 ) were generated by randomly sampling with replacement from the observed data, and the rate of statistically significant results $(p<.05)$ was estimated from the whole collection of samples. The distributions of the retention latencies were displayed as density curves in order to highlight their non-Gaussian profile and to analyze the distribution pattern specific for each experimental condition. The density curve represents the function of the probability distribution in terms of integrals. For each curve, the area under curve is equal to 1 .

\section{RESULTS}

\section{Comparison Between Single- and Double-Trial Acquisition Protocols}

The step-through latencies obtained in the single- and double-trial acquisition protocols were compared. The retention latencies of the first acquisition trial were similar in
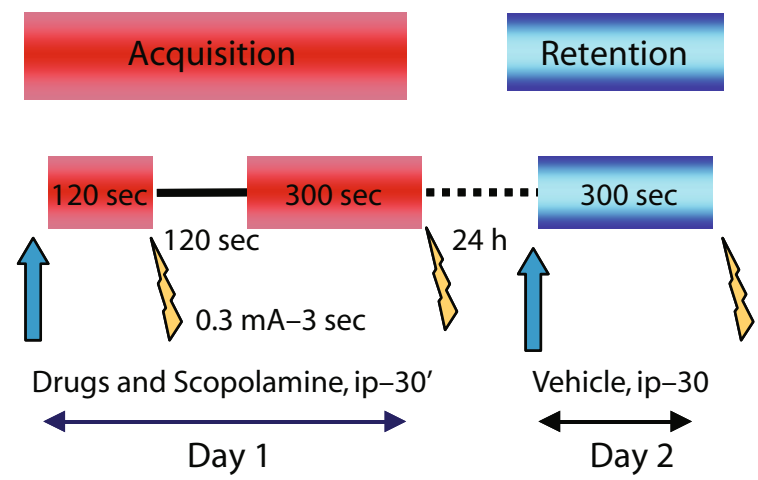

Figure 2. Double-trial acquisition protocol. 


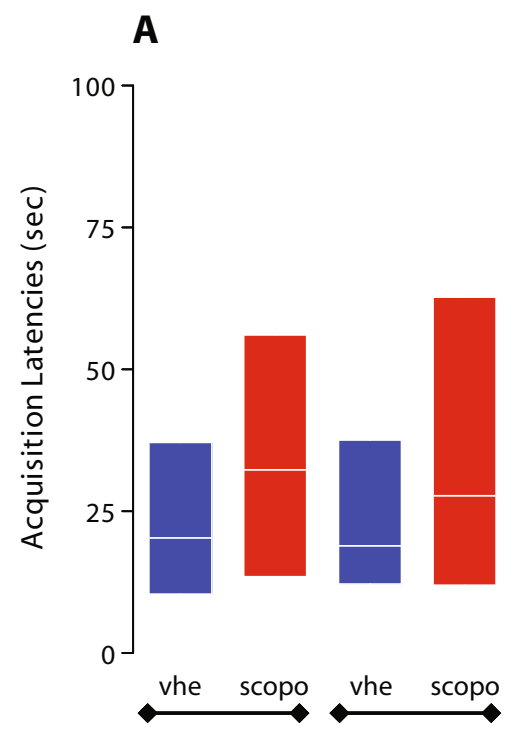

2 Acquisitions 1 Acquisition

\section{C}

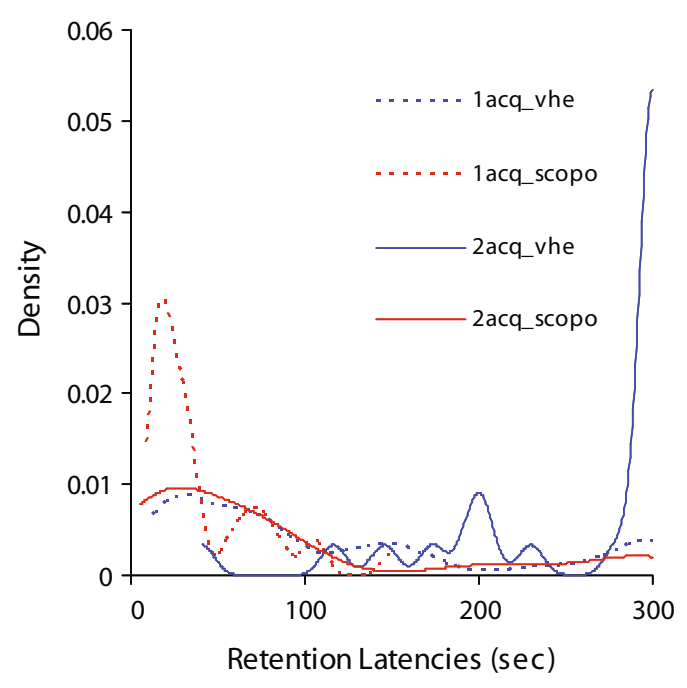

B

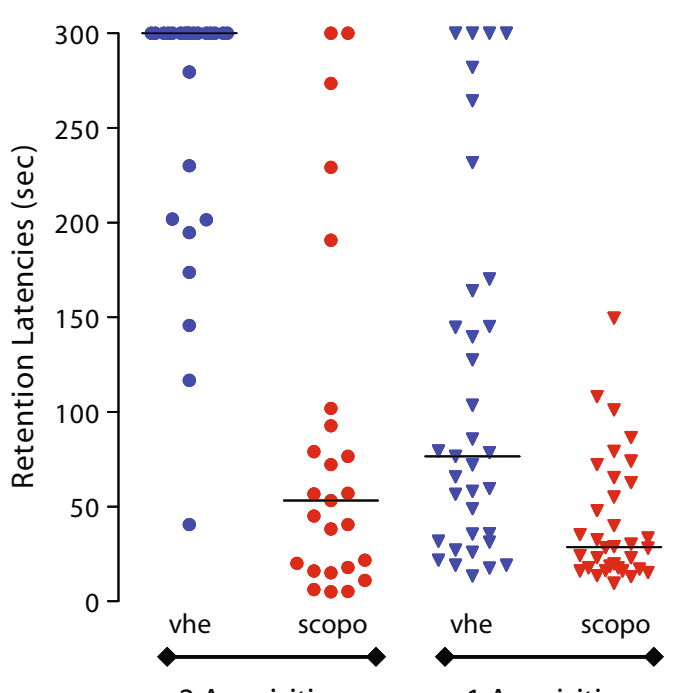

2 Acquisitions

1 Acquisition

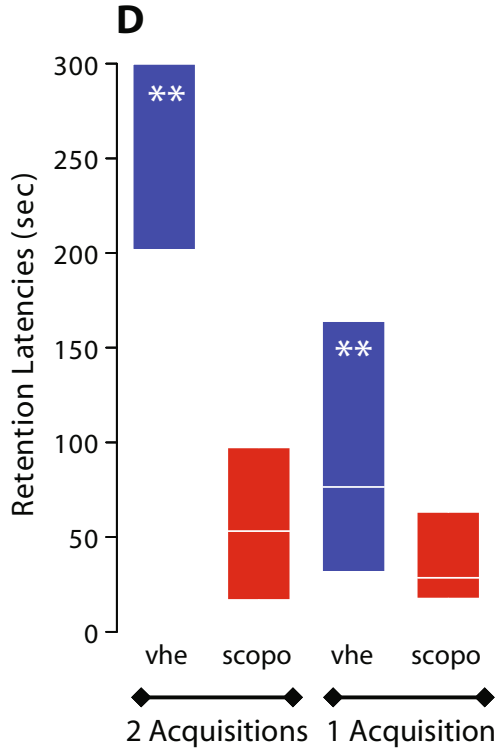

Figure 3. Comparison of the step-through latencies of the vehicle- and scopolamine-treated groups in the singleand double-trial acquisition protocols. (A) Median (Q1, Q3) of the first acquisition latency. (B) Distribution of retention latencies for vehicle- and scopolamine-treated groups in the single- and double-trial acquisition protocols. (C) Density curves of the retention latencies (1acq, single-trial acquisition; 2acq, double-trial acquisition; vhe, vehicle; scopo, scopolamine). (D) Median $(\mathrm{Q} 1, \mathrm{Q3})$ of the retention latency in both protocols. In both protocols, mice received vehicle (vhe $=\mathrm{NaCl} 0.9 \%$ ) or scopolamine $0.3 \mathrm{mg} / \mathrm{kg}$ (scopo) ip $30 \mathrm{~min}$ prior to the acquisition. Sample sizes $=\mathbf{2 5}$ and 35 for the single- and the double-trial acquisition protocol, respectively. Statistical analysis for (D): Mann-Whitney test (**p $<.01$ vhe vs. scopo).

both protocols and for both treatments, indicating similar baselines for both protocols (Figure 3A). In the double-trial acquisition protocol, most mice in the vehicle-treated group stepped through with a retention latency close or equal to the cutoff value $(300 \mathrm{sec})$. Few animals in this group had short latencies $(<100 \mathrm{sec})$ (Figure 3B). By contrast, in the single-trial acquisition protocol, the retention latencies of the vehicle-treated group were widely dispersed, with a large number of mice stepping through within $100 \mathrm{sec}$ and few mice stepping through with a latency close to $300 \mathrm{sec}$.
The distributions of retention latencies of the scopolaminetreated groups were quite similar in both protocols. Most mice stepped through in less than $50 \mathrm{sec}$ (Figure 3B). As a consequence, the smoothed histograms of distributions of retention latencies were similar in both protocols for the scopolamine-treated groups, which showed a peak density in the short-latency range (Figure 3C). By contrast, the distributions of retention latencies in the vehicle-treated groups differed in the single- and double-trial acquisition protocols (Figure 3C). The double-trial acquisition protocol showed 
A

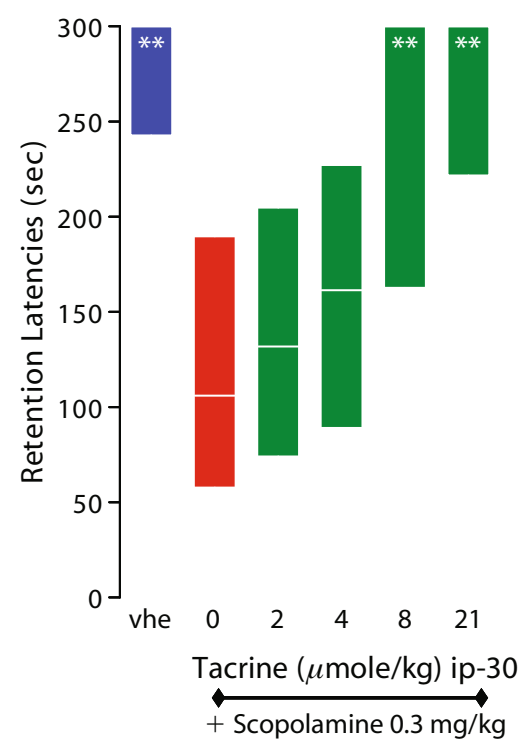

B

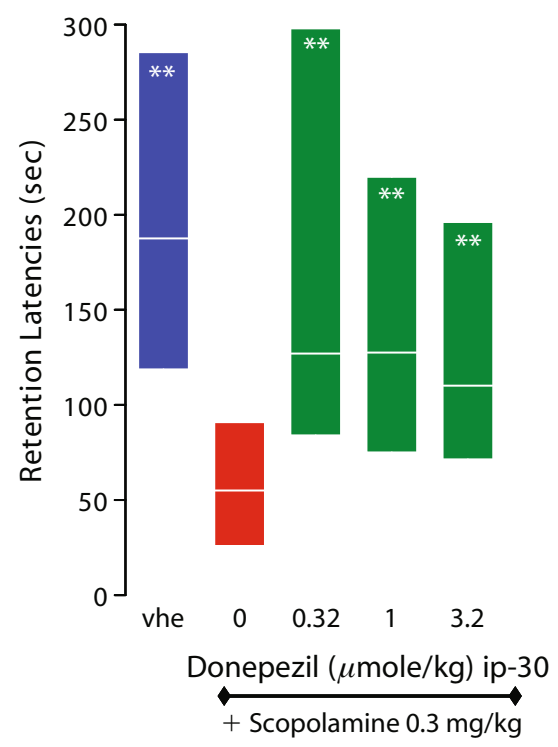

Figure 4. Pharmacological testing of the efficacy of tacrine (A) and donepezil (B) against scopolamine-induced long-term-memory deficit. Both drugs were administered ip $30 \mathrm{~min}$ prior to the first acquisition trial. Vehicle (vhe) was $0.9 \% \mathrm{NaCl}$ for tacrine and was $1 \%$ methyl cellulose-5\% DMSO for donepezil. Sample size: (A) $n=18-22$ for tacrine-treated groups and $n=30-33$ for vhe and scopolamine. (B) $n=27-28$. Statistical analysis: Mann-Whitney test versus scopolamine-treated group. ${ }^{* *} p<.01$.

a shift of density toward the highest latencies. This shift led to a clear-cut differentiation of the frequency distributions of the vehicle- versus scopolamine-treated groups. As a consequence, the overlap between the retention latencies of the vehicle- and scopolamine-treated groups disappeared in the double-trial acquisition protocol (Figure 3D). However, Mann-Whitney tests indicated that with a relatively large sample size, both protocols were equally able to display statistically significant differences between the vehicle- and scopolamine-treated groups.

\section{Reversal of the Scopolamine-Induced Memory Deficit in the Double-Trial Acquisition Protocol}

To validate the double-trial acquisition protocol for pharmacological screening of cognitive enhancers, two well-known acetylcholinesterase inhibitors - tacrine and donepezil-were tested against scopolamine (Figure 4). Tacrine is the most commonly used acetylcholinesterase inhibitor in preclinical research, whereas donepezil is the most frequently prescribed drug for Alzheimer's disease. Mann-Whitney tests performed on retention latencies indicated that tacrine significantly reversed the scopolamine-induced memory deficit at 8 and $21 \mu \mathrm{mol} /$ $\mathrm{kg}(2$ and $5 \mathrm{mg} / \mathrm{kg}$ ) to vehicle level. Donepezil at 0.32 to $3.2 \mu \mathrm{mol} / \mathrm{kg}(0.13$ to $1.3 \mathrm{mg} / \mathrm{kg})$ also significantly reduced the scopolamine-induced deficit.

\section{Reproducibility of Behavioral Response to Pharmacological Agents}

Retention latencies for vehicle, for scopolamine alone, or for scopolamine plus tacrine (obtained with the double- trial acquisition protocol) were compared during a 3-year period.

Figure 5A shows the retention latency of vehicle- and scopolamine-treated $(0.3 \mathrm{mg} / \mathrm{kg})$ groups in 11 independent experiments. In all experiments, regardless of season, the scopolamine-treated group reproducibly showed lower retention latencies than did the vehicle-treated group (statistically significant Mann-Whitney $U$ tests). For any given experiment, the distribution of the retention latencies (represented here by Q1 to Q3) was not overlapping, which confirmed the dissociation between the distributions of vehicle- and scopolamine-treated groups observed in Figure 3. These results demonstrated that the double-trial acquisition protocol was consistently and reproducibly sensitive to the scopolamine-induced impairment.

Similarly, Figure 5B shows the retention latencies of the scopolamine- $(0.3 \mathrm{mg} / \mathrm{kg})$ and scopolamine-plustacrine-treated groups in five independent experiments. Tacrine $(13 \mu \mathrm{mol} / \mathrm{kg}, 3 \mathrm{mg} / \mathrm{kg})$ reproducibly reversed the scopolamine-induced decrease of retention latencies in all five experiments (statistically significant Mann-Whitney $U$ tests).

\section{Power Analysis and Sample Size}

A power analysis was run on a pool of data from independent experiments in order to obtain a sufficiently large data set for the single- and double-trial acquisition protocols (Figure 6A). This analysis indicated that the double-trial acquisition protocol required a sample size of 5 animals per group to identify a statistical difference 
A

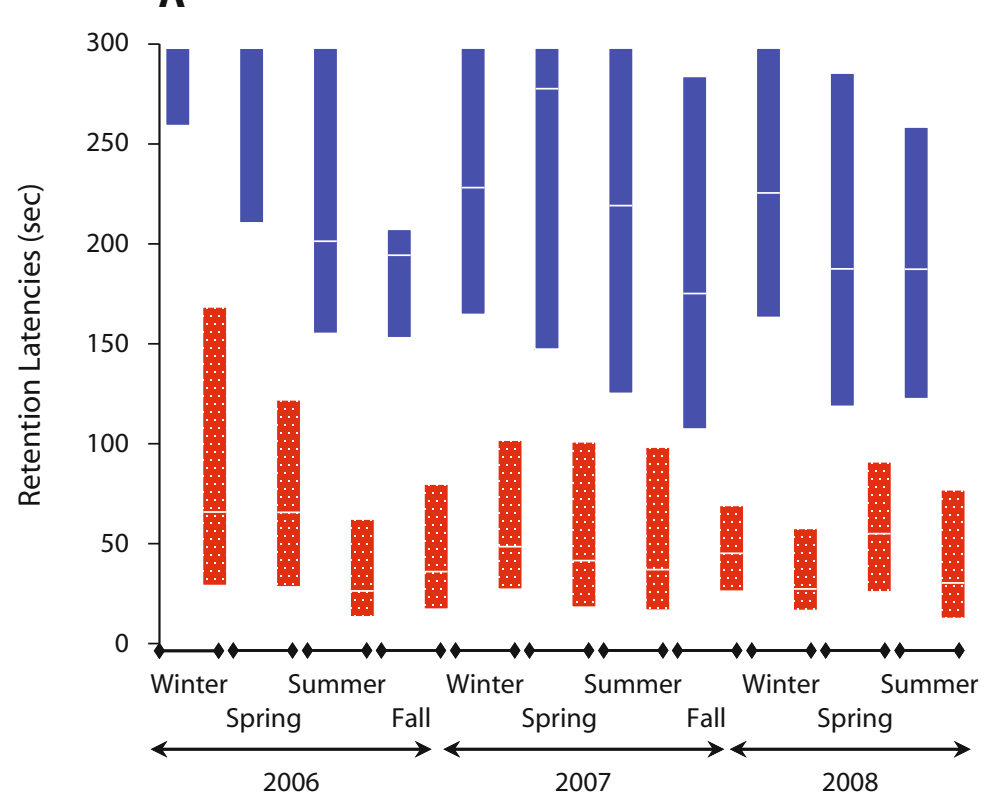

B

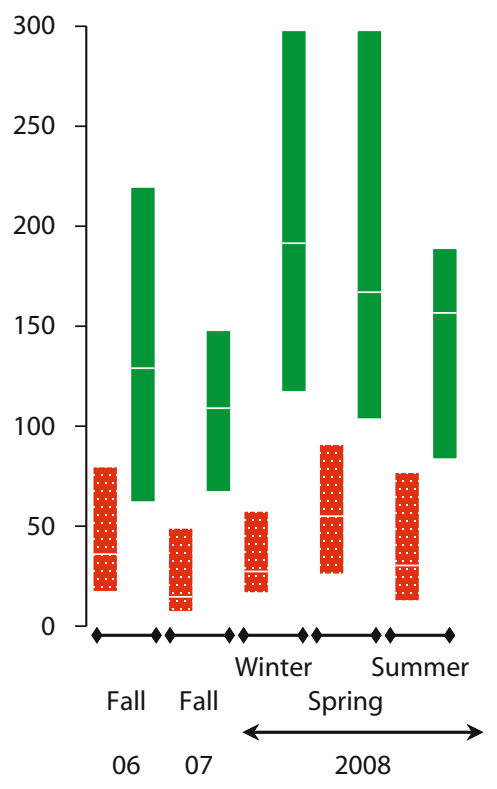

Figure 5. Reproducibility of retention latencies obtained in the double-trial acquisition protocol. (A) Comparison of retention latencies obtained in 11 independent experiments run over a 3-year period. For any of these independent experiments, the latency of vehicle-treated groups (solid columns) was higher than that of the scopolamine-treated groups (dotted columns). (Mann-Whitney tests, $p<.05$ ). (B) Comparison of scopolamine (dotted columns) versus scopolamine plus tacrine (full columns) of five independent experiments covering a 3-year period. Tacrine (13 $\mu \mathrm{mol} / \mathrm{kg}, 3 \mathrm{mg} / \mathrm{kg})$ reliably reversed scopolamine-induced decrease of retention latency. The sample size of each experiment varied from 11 to 16 per treatment. Years were divided into four periods roughly corresponding to seasons.

between the vehicle- and scopolamine-treated groups with a power of $80 \%$. A power close to $100 \%$ was obtained with a sample size of 10 . By contrast, a sample size of 15 animals per group was necessary to reach a power of $80 \%$ in the single-trial acquisition protocol. Figure 6B, which displays the density curves of retention latencies from the experiments shown in Figure 4A (dose-response curve of tacrine), clearly indicates that the frequency distribution of the tacrine-treated groups shifted from a scopolaminelike distribution to a vehicle-like distribution as the tacrine dose was increased. The scopolamine distribution showed a high density for retention around $50-70 \mathrm{sec}$, whereas the vehicle-treated group showed the highest density around 250-300 sec. The density curve for scopolamine plus
A

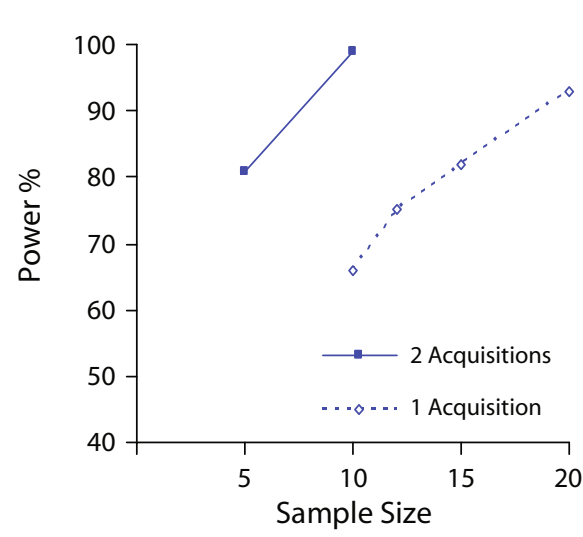

B

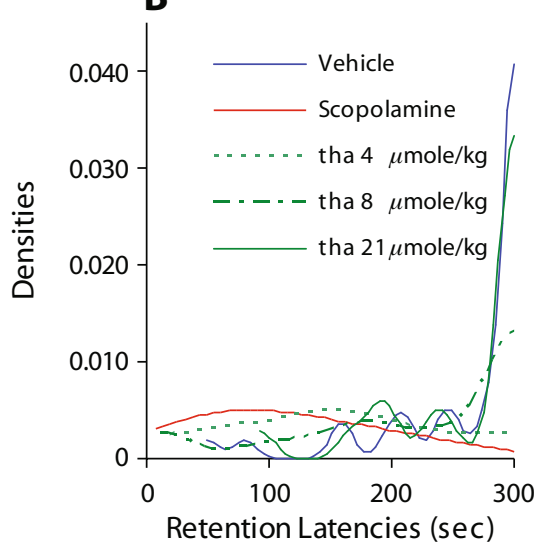

Figure 6. Power analysis and frequency distribution of retention latencies. (A) Power analysis of the single- (dotted line) and double-trial (full line) acquisition protocols, presenting the required sample size to identify a difference between scopolamine- and vehicle-treated groups with a statistical power varying between 70 and $100 \%$. (B) Density curves of retention latencies obtained in the dose-response curve of tacrine presented in Figure 4A. 2 acquisitions, doubletrial acquisition protocol; 1 acquisition, single-trial acquisition protocol; tha, tacrine. 
$21-\mu \mathrm{mol} / \mathrm{kg}$ tacrine was nearly comparable to the curve of the vehicle-treated group, whereas for scopolamine plus $4-\mu \mathrm{mol} / \mathrm{kg}$ tacrine, the density curve approached that for scopolamine alone. A statistical power analysis on these data indicated that sample sizes of 5,12 , and 80 , respectively, for the three tacrine groups would be necessary to detect a statistically significant difference at $p<.05$ with a power of $80 \%$.

\section{DISCUSSION}

Our present study aimed at improving the throughput of the inhibitory avoidance test, used as a primary behavioral screen in the discovery process of cognitive enhancers. We specifically aimed at optimizing the shortest protocol described in the literature - that is, a single-trial acquisition followed by a single retention trial. Shortly after the first acquisition trial, we added a second acquisition trial to strengthen the acquisition. We showed that the addition of a second acquisition trial separated the frequency distributions of vehicle- versus scopolamine-treated groups. These two distributions were partially overlapping in the single-trial acquisition protocol. We also demonstrated that the double-trial acquisition protocol was particularly sensitive to well-known acetylcholinesterase inhibitors such as tacrine and donepezil - drugs with demonstrated efficacy against scopolamine in this test (Dawson \& Iversen, 1993; Pan et al., 2006; Saxena, Singh, Agrawal, \& Nath, 2008; Shutske et al., 1989). Finally, we provided evidence about the reproducibility of the behavioral performance over a period of 3 years, showing reproducible scopolamine-induced long-term-memory impairments and the prevention of such a deficit by tacrine.

The choice of a specific mouse strain was of crucial importance to optimize the protocol. We chose a mouse strain with relatively low interindividual variability in the present test. C57Black6j mice were indeed ranked as good performers in this task (Crawley et al., 1997; Koide, Moriwaki, Ikeda, Niki, \& Shiroishi, 2000), and they showed a longer lasting memory to a shock and a narrower variability than did other strains (Balogh \& Wehner, 2003; Castellano \& Puglisi-Allegra, 1983; Wehner \& Balogh, 2003). We chose to administer scopolamine before the acquisition phase, since this drug has been shown to preferentially alter the acquisition process. When given after the acquisition or before the retention phase, higher scopolamine doses are usually necessary to produce memory deficits (Rush, 1988).

A power analysis of our historical data indicated that the sample size required to show statistically significant scopolamine-induced memory deficits in the double-trial acquisition protocol was half the sample size required by the single-trial acquisition protocol. The dissociation of the frequency distributions of the vehicle- and scopolamineimpaired mice explains this decrease in sample size. Moreover, this dissociation widened the window of latencies, enabling us to show a dose-sensitive reversal of the scopolamine-induced deficit. The sample size of the double-trial acquisition protocol was remarkably low in comparison with those of other protocols found in the literature. For instance, the single-trial acquisition protocol often requires sample sizes of 20-25 animals per treatment (Banfi et al., 1982).

In conclusion, we have developed a protocol for the inhibitory avoidance test that is suitable to assess the efficacy of new drugs for their potential effects against scopolamineinduced memory deficits. Our new protocol for the inhibitory avoidance test enables one to reliably cut sample size by half, which represents a marked improvement for in vivo pharmacological screening with regard to the reduction of animal use, increased throughput, and cost savings.

\section{AUTHOR NOTE}

We thank Marc De Ryck for reviewing the manuscript. Address correspondence to E.R.A.Y. Detrait, UCB, s.a., CNS Pharmacology Department-Cognitive Psychopharmacology, R9 Building, Chemin du Foriest, B-1420 Braine-l'Alleud, Belgium (e-mail: eric.detrait@ ucb-group.com).

\section{REFERENCES}

Balogh, S. A., \& Wehner, J. M. (2003). Inbred mouse strain differences in the establishment of long-term fear memory. Behavioural Brain Research, 140, 97-106.

Banfi, S., Cornelli, U., Fonio, W., \& Dorigotti, L. (1982). A screening method for substances potentially active on learning and memory. Journal of Pharmacological Methods, 8, 255-263.

Bartus, R. T., Dean, R. L., Goas, J. A., \& Lippa, A. S. (1980). Agerelated changes in passive avoidance retention: Modulation with dietary choline. Science, 209, 301-303.

BOHDANECKÝ, Z., \& JARVIK, M. E. (1967). Impairment of one-trial passive avoidance learning in mice by scopolamine, scopolamine methylbromide, and physostigmine. International Journal of Neuropharmacology, 6, 217-222.

Brunner, D., Nestler, E., \& Leahy, E. (2002). In need of highthroughput behavioral systems. Drug Discovery Today, $7(18$ Suppl.), S107-S112.

Castellano, C., \& Puglisi-Allegra, S. (1983). Strain-dependent modulation of memory by stress in mice. Behavioral \& Neural Biology, 38, 133-138.

Caulfield, M. P. (1993). Muscarinic receptors-Characterization, coupling and function. Pharmacology \& Therapeutics, 58, 319-379.

Crawley, J. N., Belknap, J. K., Collins, A., Crabbe, J. C., FranKel, W., Henderson, N., ET AL. (1997). Behavioral phenotypes of inbred mouse strains: Implications and recommendations for molecular studies. Psychopharmacology, 132, 107-124.

Dawson, G. R., \& Iversen, S. D. (1993). The effects of novel cholinesterase inhibitors and selective muscarinic receptor agonists in tests of reference and working memory. Behavioural Brain Research, 57, 143-153.

Dilts, S. L., \& Berry, C. A. (1967). Effect of cholinergic drugs on passive avoidance in the mouse. Journal of Pharmacology \& Experimental Therapeutics, 158, 279-285.

Ebert, U., \& Kirch, W. (1998). Scopolamine model of dementia: Electroencephalogram findings and cognitive performance. European Journal of Clinical Investigation, 28, 944-949.

Elrod, K., \& Buccafusco, J. J. (1988). An evaluation of the mechanism of scopolamine-induced impairment in two passive avoidance protocols. Pharmacology, Biochemistry, \& Behavior, 29, 15-21.

Graham, J. H., \& Buccafusco, J. J. (2001). Inhibitory avoidance behavior and memory assessment. In J. J. Buccafusco (Ed.), Methods of behavior analysis in neuroscience (pp. 141-151). London: CCR.

JARVIK, M. E., \& KoPP, R. (1967). An improved one-trial passive avoidance learning situation. Psychological Reports, 21, 221-224.

Kallman, M. J., \& Condie, L. W., JR. (1985). A test battery for screening behavioral teratogens in mice. Neurobehavioral Toxicology \& Teratology, 7, 727-731. 
Koide, T., Moriwaki, K., Ikeda, K., Niki, H., \& Shiroishi, T. (2000). Multi-phenotype behavioral characterization of inbred strains derived from wild stocks of Mus musculus. Mammalian Genome, 8, 664-670.

LAmberty, Y., \& Gower, A. J. (1990). Age-related changes in spontaneous behavior and learning in NMRI mice from maturity to middle age. Physiology \& Behavior, 47, 1137-1144.

Pan, S. Y., Han, Y. F., Yu, Z. L., YAnG, R., Dong, H., \& Ko, K. M. (2006). Evaluation of acute tacrine treatment on passive-avoidance response, open-field behavior, and toxicity in 17- and 30-day-old mice. Pharmacology, Biochemistry, \& Behavior, 85, 50-56.

RAY, D., \& NAGY, Z. M. (1978). Emerging cholinergic mechanisms and ontogeny of response inhibition in the mouse. Journal of Comparative \& Physiological Psychology, 92, 335-349.

Rush, D. (1988). Scopolamine amnesia of passive avoidance: A deficit of information acquisition. Behavioral \& Neural Biology, 50, 255-274.

SAHgal, A. (1993). Passive avoidance procedures. In A. Shagal (Ed.), Behavioural neuroscience: A practical approach (Vol. 1, pp. 49-56). New York: Oxford University Press.
Sarter, M., Hagan, J., \& Dudchenko, P. (1992). Behavioral screening for cognition enhancers: From indiscriminate to valid testing. Part II. Psychopharmacology, 107, 461-473.

Saxena, G., Singh, S. P., Agrawal, R., \& Nath, C. (2008). Effect of donepezil and tacrine on oxidative stress in intracerebral streptozotocin-induced model of dementia in mice. European Journal of Pharmacology, 581, 283-289.

Shutske, G. M., Pierrat, F. A., Kapples, K. J., Cornfeldt, M. L. Szewczak, M. R., Huger, F. P., ET AL. (1989). 9-Amino-1,2,3,4tetrahydroacridin-1-ols: Synthesis and evaluation as potential Alzheimer's disease therapeutics. Journal of Medicinal Chemistry, 32, 1805-1813.

WeHner, J. M., \& BAlogh, S. A. (2003). Phenotyping mice for learning and memory: Traditional tasks, modifications of traditional tasks, and application of new tasks [Society for Neuroscience, Short course].

(Manuscript received October 29, 2008;

revision accepted for publication February 6, 2009.) 